\title{
Propiedades psicométricas de la versión en castellano del Cuestionario de las Actitudes de los Estudiantes hacia la Esquizofrenia
}

\author{
Noelia NAVARRO' ${ }^{1}$, Adolfo J. CANGAS ${ }^{1}$, José M. AGUILAR-PARRA ${ }^{1}$, \\ José GALLEGO ${ }^{1}$, Emilio MORENO-SAN PEDRO², \\ Yojanán CARRASCO-RODRÍGUEZ ${ }^{2}$ y Cristina FUENTES MÉNDEZ ${ }^{2}$ \\ ${ }^{1}$ Universidad de Almería, \\ ${ }^{2}$ Universidad de Huelva
}

(Recibido, 20 Enero 2017; Aceptado, 14 Marzo 2017)

RESUMEN: En el presente estudio se analiza las propiedades psicométricas de la versión en castellano del Cuestionario de Actitudes de los Estudiantes hacia la Esquizofrenia. Se trata de un instrumento diseñado y aplicado originalmente en Alemania. Los resultados encontrados señalan un alpha de Cronbach de .95. El Análisis Factorial Exploratorio señaló la existencia, al igual que la versión original, de dos factores con una varianza total explicada del 59.94\%. Esta estructura fue confirmada por el Análisis Factorial Confirmatorio. Sin embargo, el contenido y significado de estos dos factores difieren ligeramente de la muestra alemana. En este caso, el primer factor haría referencia a miedo o temor hacia las personas con esquizofrenia, el segundo se refiere a estereotipos. Se discute la importancia de estos resultados.

Palabras clave: Estigma, Esquizofrenia, Actitudes, Discriminación, Salud Mental.

\section{Psychometric properties of the Spanish version of the questionnaire of students' attitudes towards schizophrenia}

\begin{abstract}
The present study analyzes the psychometric properties of the Spanish version of the Student Attitudes Questionnaire towards Schizophrenia. It is an instrument originally designed and applied in Germany. The results found point to a Cronbach alpha of .95. The Exploratory Factor Analysis indicated the existence, as the original version, of two factors with a total variance explained of 59.94\%. This structure was confirmed by the Factorial Confirmatory Analysis. However, the content and significance of these two factors differ slightly from the German sample. In this case, the first factor would refer to fear or fear towards people with schizophrenia, the second refers to stereotypes. The importance of these results is discussed.
\end{abstract}

Key words: Stigma, Schizophrenia, Attitudes, Discrimination, Mental Health.

Correspondencia: Noelia Navarro Gómez, Ph. D. Departamento de Psicología, Universidad de Almería, ctra. Sacramento, s/n CP04120 La Cañada de San Urbano (Almería). E-mail: nng777@ual.es 
Dentro de los trastornos psicológicos, aquellos relacionados con el espectro psicótico constituyen los que soportan mayor carga estigmatizante, algo explicable por la convergencia de diversos factores como las representaciones de la cultura popular, las imágenes dramatizadas y manipuladas por el sensacionalismo de los medios de comunicación, así como el desconocimiento y la mitología, que caricaturizan la sociedad y la inundan de estereotipos y prejuicios carentes de sustento empírico. La literatura pone de manifiesto profusamente que, al compararla con otros trastornos como la depresión, los desórdenes de ansiedad o los trastornos de la conducta alimentaria, la esquizofrenia genera una peor imagen social traducida en situaciones de miedo, desconfianza o rechazo (Wahl, 1992: Wahl, 1995; Philo, 1996).

Por todo ello, esta población puede sufrir marginación, no sólo en lo que se refiere al establecimiento de relaciones interpersonales con otros, sino también en el acceso a la vivienda, en la empleabilidad, en el tratamiento médico o en la asistencia legal. Asimismo, diversos estudios señalan que la población declara no desear que los individuos con este tipo de patología desempeñen cargos en posiciones de autoridad o poder (Pescosolido, Medina, Martin y Long, 2013).

En este sentido, diversos estudios señalan que al referirse a este colectivo suelen predominar calificativos negativos como son: incompetente, irresponsable, peligroso, impredecible, culpable de su trastorno, así como de recuperación muy difícil o imposible, a pesar de las abundantes evidencias científicas disponible en contra de estas ideas (e.g. Taylor y Dear, 1981; Hyler, Gabbard, y Schneider, 1991; Wahl, 1995; Farina, 1998; Link, Phelan, Bresnaham, Stueve, y Pescosolido, 1999; Pescosolido, Monahan, Link, Stueve, y Kikuzawa,1999; Martin, Pescosolido y Tuch, 2000; Phelan, Link, Stueve y Pescosolido, 2000).

Ahora bien, la investigación sobre el estigma relativa a la población adolescente o jóvenes no ha sido tan prolífica como en la población general. Los estudios disponibles parecen ir todos en la misma línea, señalando que es en la temprana infancia cuando se generan y desarrollan los prejuicios que posteriormente se encontrarán en los adultos. Se ha encontrado que los niños y los jóvenes parecen no tener una idea clara acerca de qué es un trastorno mental, su tratamiento o qué características estarían asociadas con el mismo (Spitzer y Cameron, 1995; Adler y Wahl, 1998; Poster, Betz, McKenna y Mossa,1986; Weiss, 1986; Poster, 1992), siendo más sofisticados estos conocimientos a medida que van creciendo. Con respecto a las actitudes estigmatizantes, la literatura arroja resultados contradictorios: desde estudios donde los participantes manifiestan niveles inicialmente bajos de estigma (Schulze et al., 2003), tienen conocimientos básicos sobre qué es la enfermedad mental y no tienen actitudes negativas hacia las personas que tienen una enfermedad mental (Ross, 2004; Watson, Ottati, Morabito, Draine, Kerr yAngell, 2010) hasta otros que muestran que los menores de 18 años sí tienen cierto tipo de prejuicios y actitudes negativas hacia las personas con trastornos mentales y por ello enfatiza que es importante realizar programas de prevención del estigma (Schachter, 2008).

La necesidad de continuar en la investigación con esta población demanda el desarrollo de instrumentos de evaluación que permitan determinar el panorama ante el que nos encontramos, esto es, el conocimiento que los jóvenes tienen de la enfermedad mental, y las personas que la padecen, así como, la presencia o no de mitos y estereotipos, para, una vez construido un perfil y determinada la necesidad de intervención, diseñar estrategias eficaces. 
En el presente estudio se muestra las propiedades psicométricas del Cuestionario de Actitudes de los Estudiantes hacia la Esquifrenia (Schulze et al., 2003). Dicho instrumento fue utilizado en una intervención escolar desarrollada en Alemania con estudiantes de secundaria, de edades comprendidas entre los 14 y los 18 años, como parte del Programa Global de la Asociación Mundial de Psiquiatría contra el estigma y la discriminación, siendo uno de los pocos instrumentos diseñados específicamente para jóvenes, no para población general. Se presentan así los resultados de una muestra española, concretamente perteneciente al Sur de España (Almería y Huelva).

\section{MÉTODO}

\section{Participantes}

En el presente estudio participaron 709 estudiantes (193 chicos y 516 chicas) de cuatro IES de las provincias de Almería y Huelva y de primer y segundo curso de las titulaciones de Educación y Psicología de las universidades de Almería y Huelva, con edades comprendidas entre los 16 y 55 años $(M=19.45, D T=3.74)$. Se utilizó un muestreo no aleatorio (Azorín \& Sánchez Crespo, 1986).

\section{Instrumento}

El Cuestionario de Actitudes de los Estudiantes hacia la Esquizofrenia (CAEE, Schulze et al., 2003). Consta de 19 ítems, divididos en dos subescalas: estereotipos de esquizofrenia (relacionados con la habilidad para gestionar el estrés, el estrato social, la intratabilidad del trastorno, la peligrosidad, la inteligencia, la impredecibilidad y la creatividad) y distancia social. Para la redacción del cuestionario original, se recurrió a la técnica de grupos focales, trabajando con jóvenes de la misma edad que los estudiantes que participaron en el estudio, lo que permitió identificar situaciones del quehacer cotidiano de los adolescentes. Con el objetivo de facilitar la comparación con otros proyectos internacionales 4 ítems de la subescala distancia social fueron tomados del proyecto piloto de la Asociación Mundial de Psiquiatría de Alberta/ Canadá. El cuestionario se validó con 90 participantes y se obtuvieron dos factores,

estereotipos y distancia social, con una alfa de Crombach para el primero de .73 y de .85 para el segundo.

\section{Procedimiento}

La versión original en inglés del CAEE se tradujo del inglés al español y de nuevo a inglés utilizando las recomendaciones propuestas por Muñiz, Elosua y Hambleton (2013). Los estudiantes fueron invitados a participar voluntariamente en esta investigación, y se requirió un consentimiento informado previo para participar. Cada aplicación llevó unos 15 minutos y se realizó en presencia del investigador principal para explicar el objetivo y la estructura de la investigación, así como la forma de cumplimentarlo. Durante dicho procesoel investigador solventó aquellas dudas que surgieron al respecto.

\section{Análisis de datos}

Se realizó un análisis inicial de las propiedades psicométricas del cuestionario. Para tal fin, se calcularon correlaciones bivariadas, análisis factorial exploratorio (AFE) y confirmatorio 
(AFC) a partir del modelo de dos factores del CAEE y un análisis de invarianza por sexo. Para realizar los análisis estadísticos se utilizó el SPSS 22 y el AMOS 21.

En primer lugar, para explorar la consistencia interna de la escala, se realizó una correlación de Pearson entre cada elemento y la puntuación total y se calculó el alfa de Cronbach para todo el conjunto de datos. En segundo lugar, se calculó el índice de KaiserMeyer-Olkin y pruebas de esfericidad de Bartlett para determinar si eran aptos para la realización del análisis factorial. Posteriormente, se desarrolló un análisis exploratorio mediante análisis de componentes principales. Finalmente, se llevó a cabo un análisis factorial confirmatorio de los ítems para ratificar el modelo obtenido en el exploratorio. Dado que para el AFC el coeficiente de Mardia resultó bajo $(215,28)$, se empleó el método de estimación de máxima verosimilitud para analizar la matriz de correlaciones. Los análisis se llevaron a cabo mediante el programa de ecuaciones estructurales AMOS.

Para tratar de aceptar o rechazar el modelo testado, se empleó una combinación de varios índices de ajuste: $\chi^{2} / g l$, CFI (Comparative Fit Index), TLI (Tucker Lewis Index), IFI (Incremental Fit Index), RMSEA (Root Mean Square Error of Approximation) más su intervalo de confianza al 90\%, y SRMR (Standardized Root Mean Square Residual). Dado que el $\chi^{2}$ es muy sensible al tamaño muestral (Jöreskog y Sörbom, 1993), se empleó el $\chi^{2} / g l$, el cual se considera aceptable, con un cociente de 4 es un ajuste razonable, mientras que aquellos valores cercanos a 2 son considerados como muy buenos (Brooke, Russell y Price, 1988).

\section{RESULTADOS}

La tabla 1 muestra las correlaciones entre cada ítem y la puntuación total de la escala que se encontró en un rango general entre.59 (ítem 18) a .84 (ítem 11). El Alpha de Cronbach global fue de .95 .

Tabla 1. Correlaciones entre cada ítem y la puntuación total de la escala. Fiabilidad.

\begin{tabular}{cccc}
\hline Items & $\begin{array}{c}\text { Correlación } \\
\text { ítem - test }\end{array}$ & $\begin{array}{c}\text { Alpha de Cronbach si } \\
\text { se elimina elemento }\end{array}$ & $\begin{array}{c}\text { Factor de saturación de cada } \\
\text { item con el principal factor }\end{array}$ \\
\hline 1 & $60^{* *}$ & .93 & .56 \\
2 & $83^{* *}$ & .93 & .68 \\
3 & $82^{* *}$ & .93 & .68 \\
4 & $66^{* *}$ & .93 & .66 \\
5 & $62^{* *}$ & .93 & .70 \\
6 & $57^{* *}$ & .93 & .66 \\
7 & $65^{* *}$ & .93 & .52 \\
8 & $76^{* *}$ & .93 & .60 \\
9 & $70^{* *}$ & .93 & .74 \\
10 & $62^{* *}$ & .93 & .77 \\
11 & $84^{* *}$ & .93 & .68 \\
12 & $77^{* *}$ & .93 & .62 \\
13 & $83^{* *}$ & .93 & .68 \\
14 & $79^{* *}$ & .93 & .71 \\
15 & $79^{* *}$ & .93 & .71 \\
16 & $81^{* *}$ & .93 & .66 \\
17 & $62^{* *}$ & .93 & .45 \\
18 & $60^{* *}$ & .93 & .80 \\
19 & $77^{* *}$ & .93 & .61 \\
\hline
\end{tabular}


En primer lugar, se desarrolló un AFE usando componentes principales con los 19 elementos que componen la escala CAEE en esta validación al castellano. En cuanto al uso del análisis factorial, la prueba de esfericidad de Bartlett debe ser estadísticamente significativa al .05 y la medida de adecuación muestral de Kaiser-Meyer-Olkin (KMO), debe ser superior a 0,6 para confirmar la aplicabilidad de la herramienta estadística (Pallant, 2001). Los datos arrojaron un coeficiente de Bartlett de 8891.06, con un $\mathrm{p}<.001$ y un KMO de .974, lo que confirma el uso del análisis factorial. Este análisis dio lugar claramente a una solución de dos factores, apoyado también en el gráfico de sedimentación (screeplot) que confirma la existencia de los mismos. Estos factores explican $59.94 \%$ de la varianza en las puntuaciones. La Tabla 1 muestra la saturación de cada ítem con el principal factor que varían entre 0,45-0,80.

Un primer AFC testó la estructura del modelo de dos factores representados por 19 ítems, revelando índices de ajuste adecuados, tal y como se aprecia en la Tabla 2. En este modelo, los pesos de regresión estandarizados fueron estadísticamente significativos $(p<, 001)$ en relación a todos los items. Los resultados de los diferentes índices de ajuste utilizados confirman que el modelo testado (dos factores correlacionados con 19 ítems) es el que mejor se ajusta a los datos (en la Figura 1 se puede observar el modelo definitivo de 19 ítems divididos en dos factores). Puede observarse que los índices incrementales (CFI, TLI e IFI) muestran un buen ajuste con valores de .90 o superiores (Schumacker y Lomax, 1996), mientras que los índices de error se consideran aceptables con valores iguales o menores de .08 para RMSEA y SRMR (Hu y Bentler, 1999). Por lo tanto, este modelo de dos factores con 19 ítems presenta un buen ajuste.

Tabla 2. Modelo de dos factores con 19 ítems para CAEE.

\begin{tabular}{llllllllllll}
\hline Modelo & $\chi^{2}$ & $g l$ & $\chi^{2} / g l$ & $p$ & N & CFI & TLI & IFI & RMSEA (IC 90\%) & SRMR \\
\hline Modelo 1 & 543,57 & 151 & 3,60 & .000 & 709 &, 955 &, 950 &, 956 &, $061(, 055-, 066)$ & \multirow{2}{*}{036} \\
\hline
\end{tabular}

Modelo 1: con 19 ítems en dos factores. 


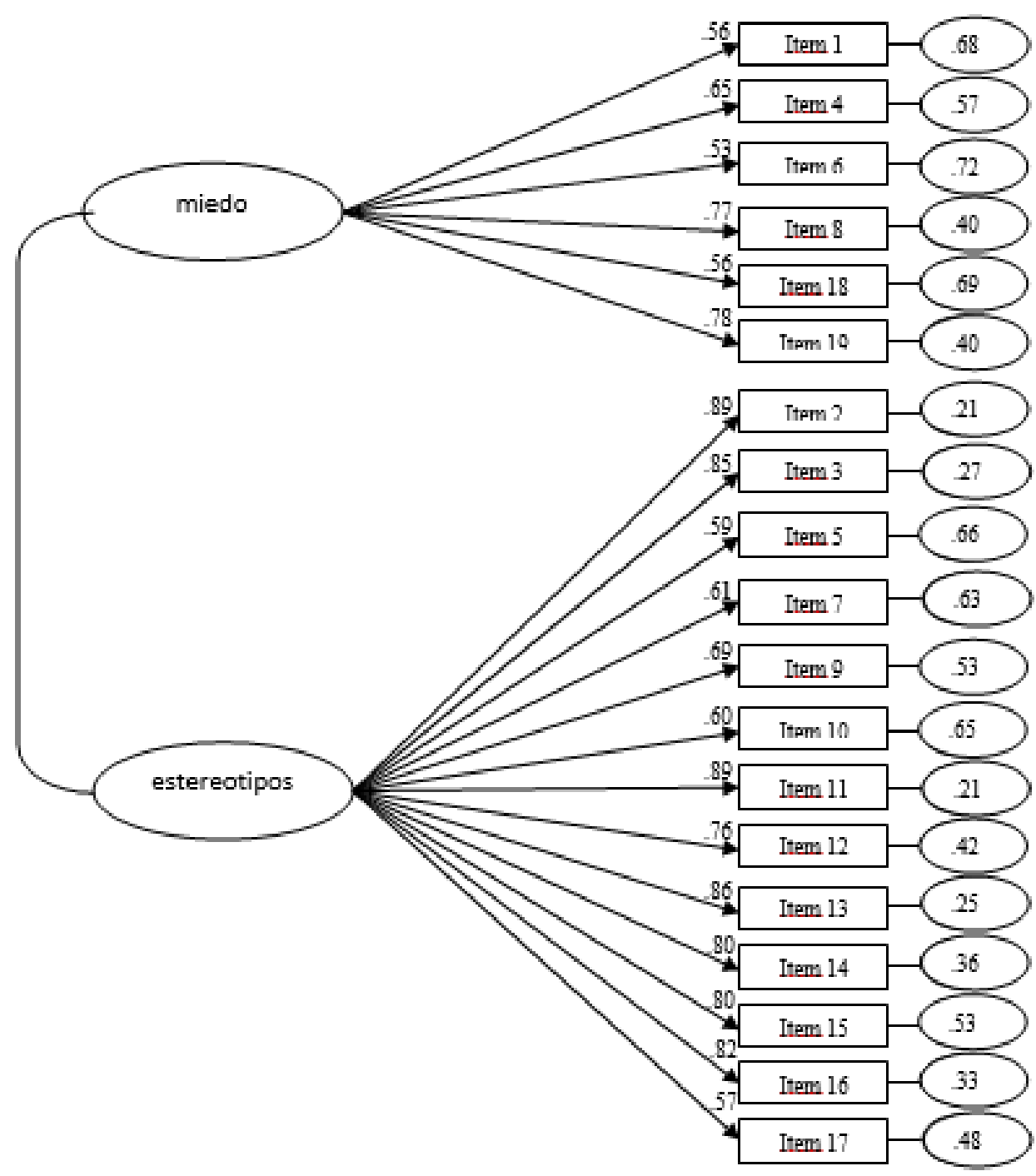

Figura 1. Modelo definitivo de 19 ítems divididos en dos factores

\section{Análisis de invarianza por sexo}

En la Tabla 3 aparecen los índices de ajuste para los cuatro modelos testados en el análisis de invarianza multigrupo respecto al género para el modelo de un factor de la escala CAEE con 19 ítems. Se verificó que no aparecen diferencias significativas en el valor de chi cuadrado entre el modelo sin restricciones y el modelo con pesos de medida, pero sí aparecen diferencias significativas al compararlo con el resto de modelos. No obstante, dado que el coeficiente $\chi^{2}$ es sensible al tamaño de la muestra, se usó el criterio establecido por Cheung y Rensvold (2002) respecto al CFI. Según estos autores, valores del CFI menores o iguales a .01 indican que la invarianza de la hipótesis nula no debe ser rechazada. De acuerdo a este criterio, los resultados apoyarían la existencia de invariancia respecto al sexo para la estructura factorial de la escala CAEE. 
Tabla 3. Análisis de Invarianza por Sexo

\begin{tabular}{llllllllllll}
\hline Modelos & $\chi^{2}$ & $g l$ & $\chi^{2} / g l$ & $\Delta \chi^{2}$ & $\Delta g l$ & CFI & TLI & IFI & RMSEA (IC 90\%) & SRMR \\
\hline Modelo 1 & 762,51 & 302 & 2,52 & - & - &, 948 &, 941 &, 948 &, $047(, 042-, 051)$ &, 052 \\
Modelo 2 & 783,67 & 319 & 2,45 & 21,15 & 17 &, 948 &, 944 &, 948 &, $045(, 041-, 050)$ &, 058 \\
Modelo 3 & 795,84 & 322 & 2,47 & $33,32 *$ & 20 &, 947 &, 943 &, 947 &, $046(, 042-, 050)$ &, 076 \\
Modelo 4 & 975,55 & 341 & 2,86 & $213,03 * *$ & 39 &, 929 &, 928 &, 929 &, $051(, 048-, 055)$ &, 089 \\
\hline
\end{tabular}

$* p<.05 ; * * p<, 01 ; * * * p<, 001$. Nota. Modelo $1=$ modelo sin restricciones; Modelo $2=$ modelo con pesos de medida invariantes; Modelo $3=$ modelo con covarianzas estructurales invariantes; Modelo $4=$ modelo con residuos de medida invariantes.

\section{DISCUSIÓN}

Los análisis estadísticos han posibilitado el análisis de las propiedades psicométicas del Cuestionario de Actitudes de Estudiantes hacia la Esquizofrenia (Schulze et al., 2003). En efecto, el instrumento mantiene propiedades psicométricas similares al original al aplicarse en nuestro país con población adolescente. Así, se ha mantenido la estructura bifactorial, aunque han cambiado ligeramente la distribución y el contenido de los mismos: en la validación a nuestra muestra, el factor distancia social, se correspondería con el factor, "miedo", mientras que el factor "estereotipos", se mantiene, aunque cambiando los ítems, con respecto al cuestionario aplicado en población alemana.

Es quizá la consideración de violencia lo que más predomina al hablar de un trastorno mental grave como es la esquizofrenia (López, Laviana, Fernández, López, Rodríguez, y Aparicio, 2008; Angermeyer y Dietrich, 2006; Angermeyer y Matschinger, 1996; Walsh, Buchanan y Fahy, 2002) de ahí que quizá por eso este factor ha sido predominantes en el análisis del cuestionario, agrupándose en un factor ítems que hacen referencia a este hecho de temor como, por citar algunos ejemplos, "cuando quedas con alguien con esquizofrenia, hay que andarse con cuidado", "alguien con esquizofrenia puede ponerse furioso por una tontería" o "tendría miedo de hablar con una persona con esquizofrenia",

De hecho, en el caso de la enfermedad mental los comportamientos relacionados con la percepción de peligrosidad emergen (Brockington, Hall, Levings y Murphy, 1993; Link, et al., 1999). El factor miedo es común a diversos estudios que evalúan las actitudes de los estudiantes afirmando algunos participantes tener miedo o sentirse incómodos ante aquellos estudiantes con enfermedad mental (Becker, Martin, Wajeeh, Ward y Shern, 2002), siendo esta una variable predictora del mal funcionamiento en la integración de aquellos diagnosticados con dicha etiqueta. En efecto, la percepción de peligrosidad, con el consiguiente miedo que esta conlleva, desemboca finalmente en conductas aprensivas o de evitación (Corrigan y Watson, 2002), así como, de forma consistente con la distancia social, componente de peso en el proceso de estigmatización (Link et al.,1999).

Los estereotipos compondrían el segundo factor, que indirectamente podrían conducir a una baja implicación de los demás en la interacción con estas personas, y estaría conformado por ítems como, "en la mayoría de los casos, alguien con esquizofrenia viene de una familia de 
poco dinero", "alguien con esquizofrenia no puede ser ayudado por los médicos" o "alguien con esquizofrenia puede ser bueno en los estudios". De acuerdo con la lógica del proceso de formación del estigma, las características indeseables que conforman los estereotipos se vincularían a una determinada condición y servirían como justificación para determinadas intenciones conductuales (Link y Phelan, 2001).

Si bien, aunque se mantienen la estructura bifactorial, el factor distancia social, ha sido reemplazado en nuestro análisis por el factor estereotipos, concepto más amplio, y que, en cualquier caso, podría igualmente conducir a situaciones de marginación y exclusión. Asimismo, se observa que varían los ítems que componen cada uno de estos dos factores, con respecto al cuestionario original. Esta diferencia en los ítems puede venir influenciada también por el análisis llevado a cabo al instrumento. Recordemos que el cuestionario original fue construido mediante la metodología de grupo focal, con una muestra de 90 estudiantes de la misma edad, con el objetivo de identificar situaciones relevantes de su vida cotidiana, tomándose además 4 ítems de distancia social de otro proyecto piloto desarrollado en Alberta (Canadá). Sin embargo, en este caso se ha llevado a cabo un análisis factorial tanto exploratorio como confirmatorio con una muestra significativamente mayor. De ahí que las diferencias puedan deberse también a las diferencias en la metodología utilizada.

De este modo, se constata la utilidad del cuestionario para utilizar en nuestra cultura, tanto como herramienta para conocer las actitudes de los estudiantes, como para, comprobar la efectividad de las intervenciones antiestigma en población adolescente, reflejando, caso que lo hubieran, los cambios en las actitudes y creencias hacia este trastorno.

\section{AGRADECIMIENTOS}

El presente trabajo fue financiado por un proyecto de Excelencia de la Junta de Andalucía concedido al segundo autor (SEJ-7204).

\section{REFERENCIAS}

Adler, A. K. y Wahl, O. F. (1998). Children's beliefs about people labeled mentally ill. American Journal of Orthopsychiatry, 68, 321-326.

Angermeyer, M. y Dietrich, S. (2006). Public beliefs about and actitudes towards people with mental illness: a review of population studies. Acta Psychiatrica Scandinavica, 113, 163-179.

Angermeyer, M. y Matschinger, H. (1996). The effect of violent attacks by schizophrenic persons on the attitude of the public towards the mentally ill. Social Science \& Medicine, 43,1721-1728.

Azorín, F. y Sánchez-Crespo. (1986). Métodos y aplicaciones del muestreo. Madrid: Alianza Editorial.

Becker, M., Martin, L., Wajeeh, E., Ward, J. y Shern, D. (2002). Students with mental illnesses in a universitysetting: faculty and student altitudes, beliefs, knowledge, and experiences. Psychiatric Rehabilitation Journal, 25, 359-368.

Brockington, I. F., Hall, P., Levings, J. y Murphy, C. (1993). The community's tolerance of the mentally ill. The British Journal of Psychiatry,162, 93-99. 
Brooke, P. P., Russell, D. y Price, J. L. (1988). Discriminant validation of measures of job satisfaction, job involvement, and organizational commitment. Journal of Applied Psychology, 73, 139-145.

Cheung, G. W. y Rensvold, R. B. (2002). Evaluating goodness-of-fit indexes for testing measurement in variance. Structural Equation Modeling, 9, 233-255.

Corrigan, P. W. y Watson, A. C. (2002). Understanding the impact of stigma on people with mental illness. World Psychiatry; Official Journal of the World Psychiatric Association (WPA), 1, 16-20.

Farina, A. (1998). Stigma. En K. T. Muesser, y N. Tarrier (Ed.), Handbook of Social Functioning in Schizophrenia (pp. 247-279). Boston: Allyn and Bacon.

Hu, L. y Bentler, P. M. (1999). Cutoff Criteria for Fit Indexes in Covariance Structure Analysis: Conventional Criteria Versus New Alternatives. Structural Equation Modeling, 6,1-55.

Hyler, S. E., Gabbard, G. O. y Schneider, I. (1991). Homicidal maniacs and narcissistic parasites: Stigmatization of mentally ill persons in the movies. Hospital \& Community Psychiatry, 42, 1044-1048.

Jöreskog, K. G. y Sörbom, D. (1993). LISREL 8: Structural equation modeling with the SIMPLIS command language. Hillsdale, Nueva Jersey: Lawrence Erlbaum Associates Publishers.

Link, B. G., Phelan. J. C., Bresnahan, M., Stueve, A. y Pescolido, B. A. (1999). Public conceptions of mental illness: labels, causes, dangerousness, and social distance. American Journal of Public Health, 89, 1328-1333.

Link, B. G. y Phelan J. C. (2001). Conceptualizing Stigma. Annual Review of Sociology,27, 363-385.

López, M., Laviana, M., Fernández, L., López, A., Rodríguez, A. M. y Aparicio, A. (2008). La lucha contra el estigma y la discriminación en salud mental. Una estrategia compleja basada en la información disponible. Revista de la Asociación Española de Neuropsiquiatría, 101, 43-83.

Martin, J. K., Pescosolido, B. A. y Tuch, S. A. (2000). Of fear and loathing: The role of "disturbing behavior", labels, and causal attributions in shaping public attitudes toward people with mental illness. Journal of Health and Social Behavior, 41, 208-223.

Muñiz, J., Elosou, P. y Hambleton, R. K. (2013). Directrices para la traducción y adaptación de los tests: segunda edición. Psicothema,25, 151-157.

Ottati, V., Bodenhausen, G. y Newman, L. (2005). Social psychological models of mental illness stigma. En P. W. Corrigan (Ed.), On the Stigma of mental illness: Practical strategies for research and social change (pp. 99-128). Washington, DC: American Psychological Association.

Pallant, J. (2001). The SPSS survival manual: A step-by-step guide to data analysis using SPSS for Windows (version 10). St. Leonards, NSW: Allen \&Unwin.

Pescosolido, B. A., Monahan, J., Link, B. G., Stueve, A. y Kikuzawa, S. (1999). The public's view of the competence, dangerousness and need for legal coercion of persons with mental health problems. American Journal of Public Health, 89, 1339-1345. 
Pescosolido, B. A., Medina, T. R., Martin, J. K. y Long, J. S. (2013). The "backbone" of stigma: identifying the global core of public prejudice associated with mental illness. American Journal of Public Health, 103, 853-860.

Phelan, J. C., Link, B. G., Stueve, A. y Pescosolido, B. A. (2000). Public conceptions of mental illness in 1950 and 1996: What is mental illness and is it to be fear? Journal of Health and Social Behavior, 41, 188-207.

Philo, G. (1996). Media and mental distress. London y New York: Longman.

Poster, E. C., Betz, C., McKenna, A. y Mossar, M. (1986). Children's attitudes toward the mentally ill as reflected in human figure drawings and stories. Journal of the American Academy of Child Psychiatry, 25, 680-686.

Poster, E. C. Children's concepts of thementallyill. (1992). Journal of Adolescent Pediatric and Mental Health Nursing, 5, 28-36.

Ross, M. (2004). An evaluation of anti-stigma and educational program in Hamilton Ontario. Recuperado el 17 de marzo de 2017, http://www.bridgeross.com/TAMIdraft.pdf.

Schachter, H. M., Girardi, A., Ly, M., Lacroix, D., Lumb, A. B., van Berkom, J. y Gill, R. (2008). Effects of school-based interventions on mental health stigmatization: a systematic review. Child and Adolescent Psychiatry and Mental Health, 2,18-32.

Schulze, B., Richter-Werling, M., Matschinger, H. y Angermeyer, M. C. (2003). Crazy? So what! Effects of a school project on students' attitudes toward people with schizophrenia. Acta Psychiatrica Scandinavica, 107,142-150.

Schumacher, R. E. y Lomax, R. G. (1996). A Beginner's Guide to Structural Equation Modeling. New Jersey: Lawrence Erlbaum Associates, Publishers.

Spitzer, A. y Cameron, C. (1995). School-agechildren's perceptions of mental illness. Western Journal of Nursing Research, 17, 398-415.

Taylor, S. M. y Dear, M. J. (1981). Scaling community attitudes toward the mentally ill. Schizophrenia Bulletin, 7, 225-240.

Wahl O. (1992). Mass media images of mental illness: a review of the literature. Journal of Community Psychology, 20,343-352.

Wahl O. (1995). Media madness. Public images of mental illness. New Brunswick, Nueva Jersey: Rutgers University Press.

Walsh, E., Buchanan, A. y Fahy, T. (2002). Violence and schizophrenia: examining the evidence. British Journal of Psychiatry, 180, 490-495.

Watson, A. C., Ottati, V. C., Morabito, M. S., Draine, J., Kerr, A. N. y Angell, B. (2010). Outcomes of police contacts with persons with mental illness: The impact of CIT. Administration and Policy in Mental Health and Mental Health Services Research, 37,302-317.

Weiss, M. F. (1986). Children's attitudes toward thementally ill: A developmental analysis. Psychological Reports, 58, 11-20. 